\title{
Geometric Operators in the Einstein-Hilbert Truncation
}

\author{
Maximilian Becker ${ }^{\dagger}$ and Carlo Pagani ${ }^{*, \dagger}$ \\ Johannes Gutenberg University Mainz, Staudingerweg 7, D-55099 Mainz, Germany; bemaximi@uni-mainz.de \\ * Correspondence: capagani@uni-mainz.de; Tel.: +49-6131-3927253 \\ + These authors contributed equally to this work.
}

Received: 14 February 2019; Accepted: 5 March 2019; Published: 11 March 2019

\begin{abstract}
We review the study of the scaling properties of geometric operators, such as the geodesic length and the volume of hypersurfaces, in the context of the Asymptotic Safety scenario for quantum gravity. We discuss the use of such operators and how they can be embedded in the effective average action formalism. We report the anomalous dimension of the geometric operators in the Einstein-Hilbert truncation via different approximations by considering simple extensions of previous studies.
\end{abstract}

Keywords: asymptotic safety; geometric operators; functional renormalization group

\section{Introduction}

In the Asymptotic Safety (AS) scenario, gravity is quantized in a quantum field theoretic framework [1]. In this approach a fundamental quantum field theory of gravity is defined thanks to the presence of an ultraviolet-attractive non-Gaussian fixed point with a finite number of relevant directions $[1,2]$. Thanks to such a fixed point the theory is predictive up to arbitrarily small length scales.

The AS fixed point has non-perturbative nature and to probe its existence one must resort to non-perturbative techniques. A promising approach to investigate such a research line has been laid down in [2] by Martin Reuter. In [2] a functional renormalization group (FRG) flow equation for quantum gravity was derived in terms of the so-called effective average action (EAA) [3], which is a scale dependent generalization of the usual effective action. The EAA realizes the Wilsonian renormalization program by suppressing the momentum modes below a scale $k$. Furthermore, the EAA interpolates between the microscopic action $\left(k \rightarrow \infty \operatorname{limit}^{1}\right)$ and the quantum effective action $(k \rightarrow 0$ limit). Thus, by lowering the scale $k$, one can perform a piecemeal functional integral for quantum gravity that can be employed to make actual predictions.

From a more phenomenological viewpoint, quantum gravity effects are supposed to be significant from energies higher than the Planck mass so that natural places to look for such effects are the cosmology of the early universe and black hole physics. The AS scenario for quantum gravity has opened new possibilities in these fields and led to new insights, (see e.g., [5-10]).

Even if the scale dependence of the EAA is governed by an exact equation $[3,11,12]$, the latter can be solved by implementing some approximation scheme. The gravitational extension of the EAA allows one to use this framework to investigate the presence of a suitable fixed point for the AS scenario for quantum gravity [2]. Strong evidence for the presence of such a fixed point has been found in a large number of approximation schemes. In particular, the AS fixed point has been consistently found in various types of truncations, which include higher curvature terms [13-20], the Goroff-Sagnotti counterterm [21], and polynomials of the Ricci scalar of high order [22-25]. Furthermore, approximation

1 See [4] for a more detailed discussion on this point. 
schemes involving infinite dimensional truncations have been studied [26-40]. Finally, also ansätze that take into account the bimetric character of the EAA have been investigated in various settings [41-51].

However, even in the presence of a suitable fixed point, further effort is often needed to make contact with gravitational observables [52,53]. The reason for this is that on top of the EAA, one needs to make contact with geometric operators such as the geodesic length. (See [52] for a discussion in the AS framework.) Such geometric operators are not generally present in any truncation for the EAA, which is built as a functional of (quasi)local operators. To circumvent this difficulty, one can consider geometric operators as composite operators, which are renormalized over and above the standard EAA.

In this work we review the study of certain geometric operators and report the scaling properties of geodesics and the volume of hypersurfaces by considering simple extensions of previous works. The paper is organized as follows. In Section 2 we introduce and discuss the geometric operators of interest. In Section 3 we discuss the Einstein-Hilbert truncation and how to compute the scaling properties of geometric operators. In Section 4 we report the values of the anomalous dimensions of the geometric operators. Finally, we summarize the paper in Section 5.

\section{Geometric Operators in Asymptotic Safety}

In this section, we introduce the geometric operators that we study in this work and outline their possible use in the AS scenario for quantum gravity.

\subsection{Volume of Hypersurfaces in Spacetime}

Let us consider the volume of an $n$-dimensional submanifold, which we denote by $\sigma_{n}$ and that is embedded into the $d$-dimensional spacetime manifold $M$. Let us also assume that $\sigma_{n}$ can be covered by a single chart. The $n$-dimensional hypersurface $\sigma_{n}$ is parametrized by $n$ coordinates, which we denote by $u^{a}$, with $a=1, \cdots, n$. The hypersurface is then characterized by a map $x^{\mu}(u)$. The induced metric on $\sigma_{n}$ is given by the pullback of the manifold metric, that is

$$
g_{a b}(u)=g_{\mu v}(x(u)) \frac{\partial x^{\mu}(u)}{\partial u^{a}} \frac{\partial x^{v}(u)}{\partial u^{b}},
$$

where $g_{a b}$ is the metric on $\sigma_{n}$. The volume of the submanifold $\sigma_{n}$ can then be written as

$$
V_{\sigma_{n}} \equiv \int_{\sigma_{n}} \sqrt{x_{* g}}=\int_{U} d^{n} u \sqrt{\operatorname{det} g(u)}
$$

As an example, for $n=1$ Equation (2) reproduces the length of a given curve $x^{\mu}(u)$ :

$$
V_{\sigma_{1}}=\int d u \sqrt{g_{\mu v}(x(u)) \dot{x}^{\mu}(u) \dot{x}^{v}(u)} .
$$

Dealing with such geometric objects, it is natural to ask what kind of behavior the volume $V_{\sigma_{n}}$ possesses at the quantum level. Let us assume that the volume is characterized by an externally prescribed length $L$. At the classical level we expect that $V_{\sigma_{n}} \sim L^{n}$. However, quantum fluctuation may change this expectation, so that on general grounds one imagines

$$
\left\langle V_{\sigma_{n}}\right\rangle \sim L^{n-\gamma_{\sigma_{n}}},
$$

where $\gamma_{\sigma_{n}}$ is an anomalous dimension associated with $V_{\sigma_{n}}$. The (approximate) computation of $\gamma_{\sigma_{n}}$ will be given in Section 4. Given the scaling (3) one can also express the scaling of $\left\langle V_{\sigma_{n}}\right\rangle$ via $\ell \equiv\left\langle V_{\sigma_{1}}\right\rangle$ :

$$
\left\langle V_{\sigma_{n}}\right\rangle \sim \ell^{\frac{n-\gamma_{\sigma_{n}}}{1-\gamma_{\sigma_{1}}}} .
$$


Even if the averages (3) and (4) are not diffeomorphism invariant observables, one may suspect that such geometric quantities still carry certain features of AS. In this respect, we shall see that explicit computations give $\gamma_{\sigma_{n}}>0$. From such values of the anomalous dimension and the relation $\left\langle V_{\sigma_{n}}\right\rangle \sim L^{n-\gamma_{\sigma_{n}}}$ one infers that AS typically implies an effective dimensional reduction of the scaling properties of spacetime. Similar dimensional reduction phenomena are a feature common to several quantum gravity scenarios [54].

\subsection{The Geodesic Length}

As mentioned in the introduction, to make contact with diffeomorphism invariant observables one has often to resort to non-local operators. As an example of such an observable, let us consider the correlation function of two operators at fixed geodesic length. This is defined by (see e.g., $[55,56])$

$$
G(r)=\left\langle\int d^{d} x \sqrt{g(x)} \int d^{d} y \sqrt{g(y)} O_{1}(x) O_{2}(y) \delta\left(r-\ell_{g}(x, y)\right)\right\rangle,
$$

where $\ell_{g}(x, y)$ is the geodesic length. We will define $\ell_{g}(x, y)$ precisely later. For the time being, we wish to emphasize that the correlation function defined in (5) crucially depends also on the geodesic length, which is an operator that does not appear in any truncation for the gravitational EAA.

Along lines similar to those reported in [57], let us now provide an argument that allows one to deduce the scaling behavior of $G(r)$. This argument has the purpose to show the key role played by the scaling properties of the operators involved in the definition (5), including the geodesic length. We have

$$
\begin{aligned}
G_{12}(\lambda r) & =\left\langle\frac{1}{\operatorname{Vol}} \int_{x, y} \sqrt{g_{x}} O_{1}(x) \sqrt{g_{y}} O_{2}(y) \delta\left(\ell_{g}(x, y)-\lambda r\right)\right\rangle \\
& =\frac{1}{Z} \int \mathcal{D} g e^{-S} \int_{x, y} \frac{1}{\operatorname{Vol}} \sqrt{g_{x}} O_{1}(x) \sqrt{g_{y}} O_{2}(y) \delta\left(\ell_{g}(x, y)-\lambda r\right) \\
& =\left\langle\int_{x, y} \frac{\Omega^{\Delta_{\mathrm{Vol}}^{g}}-\Delta_{1}^{g}-\Delta_{2}^{g}}{\mathrm{Vol}} \sqrt{g_{x}} O_{1}(x) \sqrt{g_{y}} O_{2}(y) \delta\left(\Omega^{-\Delta_{d_{g}}^{g} \ell_{g}}(x, y)-\lambda r\right)\right\rangle \\
& =\left\langle\lambda \frac{\Delta_{1}^{g}+\Delta_{2}^{g}-\Delta_{\mathrm{Vol}}^{g}}{\Delta_{\text {dg }}^{g}} \int_{x, y} \sqrt{g_{x}} O_{1}(x) \sqrt{g_{y}} O_{2}(y) \delta\left(\lambda \ell_{g}(x, y)-\lambda r\right)\right\rangle \\
& =\lambda \frac{\Delta_{1}^{g}+\Delta_{2}^{g}-\Delta_{\mathrm{Vol}}^{g}-1}{\Delta_{d g}^{g}} G_{12}(r),
\end{aligned}
$$

where $Z$ is the partition function, and Vol is the volume operator, i.e., $\int \sqrt{g}$. Setting $\lambda=r^{-1}$ in Equation (6) one obtains the scaling behavior of the correlation function $G(r)$. Let us spell out the steps that lead to Equation (6). After having rewritten the average via a path integral in the second line, we performed a dummy change of variables. In particular, we performed a scale transformation characterized by a parameter $\Omega$ and assumed being in the fixed-point regime, where all operators transform homogeneously under a scale transformation. The parameter $\Omega$ is then chosen to satisfy $\Omega^{-\Delta_{d g}^{g}}=\lambda$ so that the $\lambda$ can be pulled out of the functional Dirac delta.

A few comments are in order. First, we wish to emphasize that the above argument is a qualitative one and that it may not be complete. In particular, we implicitly assumed a scale invariant measure of the path integral. However, the transformation properties of the measure are known to play an important role in two-dimensional gravity, see $[52,57,58]$ for an FRG perspective on these issues. Moreover, it must be emphasized that the mass dimensions $\Delta_{i}$ are the full quantum scaling dimensions of the operators involved. This implies that $\Delta_{i}$ are not the classical (engineering) dimensions, but that the classical scaling is corrected by an anomalous dimension. This latter anomalous dimension must be computed explicitly, even for non-local operators like the geodesic length. 
There are also further geometric operators that encode information about the structure of spacetime. Of particular interest is the volume of a geodesic ball since its scaling $\left\langle V_{\text {ball }}(r)\right\rangle \sim r^{d_{\mathrm{H}}}$ is characterized by the Hausdorff dimension $d_{\mathrm{H}}$ of the spacetime, (see e.g., [59]).

In Sections 3 and 4 we show that both the scaling properties of the geodesic length and the volume of hypersurface can be studied by suitably extending the EAA.

\section{The Effective Average Action and Geometric Operators}

\subsection{The Einstein-Hilbert Truncation for the EAA}

In the EAA formalism one realizes the Wilsonian renormalization program by introducing a scale $k$ below which the integration of momentum modes is suppressed. Such suppression is implemented by adding to the classical action the so-called cutoff action $\Delta S_{k}$. The cutoff action is quadratic in the quantum fields $\chi$ and is characterized by a suitable kernel $\mathcal{R}_{k}$, i.e., $\Delta S_{k}[\chi]=\frac{1}{2} \int \chi \mathcal{R}_{k} \chi$.

One can then introduce the modified generating functional for the connected correlation functions $W_{k}[J]$ via

$$
e^{W_{k}[J]} \equiv \int \mathcal{D} \chi e^{-S[\chi]-\Delta S_{k}[\chi]+J \cdot \chi}
$$

The EAA is then defined as the Legendre transform of $W_{k}[J]$ after the subtraction of the cutoff term, i.e., by $\Gamma_{k}[\varphi]=J \cdot \varphi-W_{k}[J]-\Delta S_{k}[\varphi] .^{2}$ The scale dependence of the EAA is governed by the following exact equation $[3,11,12]$

$$
\partial_{t} \Gamma_{k}=\frac{1}{2} \operatorname{Tr}\left[\left(\Gamma_{k}^{(2)}+\mathcal{R}_{k}\right)^{-1} \partial_{t} \mathcal{R}_{k}\right],
$$

where $\Gamma_{k}^{(2)}$ denotes the Hessian of the effective average action and $t \equiv \log k$.

Equation (8) can be solved after implementing some approximation scheme, such as expanding the EAA in a finite number of monomials:

$$
\Gamma_{k} \equiv \sum_{i=1}^{m} g_{i} M_{i}
$$

where $M_{i} \in\left\{\int \sqrt{g}, \int \sqrt{g} R, \cdots\right\}$. In this work we shall consider the so-called Einstein-Hilbert truncation, which is defined by

$$
\Gamma_{k}[g]=\frac{1}{16 \pi G_{k}} \int d^{d} x \sqrt{g}\left(2 \Lambda_{k}-R\right) .
$$

Employing the background field method, the ansatz (9) is equipped with a gauge-fixing term and the associated ghosts. In particular we will work in the Feynman-de Donder gauge, (see e.g., [16]). Note also that (9) is a single metric ansatz, which is defined as a functional of the metric $g_{\mu v}=\bar{g}_{\mu v}+h_{\mu \nu}$ with $\bar{g}_{\mu \nu}$ being the background metric and $h_{\mu \nu}$ being the average fluctuating metric. In full generality, however, the EAA is expected to be a generic functional of $\bar{g}_{\mu v}$ and $h_{\mu v}$ due to the breaking of the split symmetry, i.e., $\Gamma_{k}[\bar{g}, h]$.

\subsection{Geometric Operators as Composite Operators}

We now consider the following situation. Let us suppose that we have been able to compute the EAA within a certain approximation. We ask ourselves how one could extract information regarding operators not included in the EAA. A possible way to tackle this situation is to consider an extended

2 From here on the dot denotes the DeWitt summation and integration convention, e.g., $J \cdot \varphi=\int_{x} J_{i}(x) \varphi^{i}(x)$. 
version of the EAA in which new sources conjugated to the operators of interest are introduced. More precisely one defines the following modified generating functional $W_{k}[J, \varepsilon]$ via

$$
e^{W_{k}[J, \varepsilon]} \equiv \int \mathcal{D} \chi e^{-S[\chi]-\Delta S_{k}[\chi]+J \cdot \chi-\varepsilon \cdot O}
$$

Equation (10) differs from (8) only in the last term of the argument of the exponential. The EAA is then defined as usual via $\Gamma_{k}[\varphi, \varepsilon]=J \cdot \varphi-W_{k}[J, \varepsilon]-\Delta S_{k}[\varphi]$, where no Legendre transform has been implemented on the sources $\varepsilon_{i}(x)$.

By taking functional derivatives with respect to the source $\varepsilon_{i}(x)$ one obtains information regarding the insertion of a composite operator:

$$
\left\langle O_{i}(x)\right\rangle_{J}=-\left.\frac{\delta W_{k}[J, \varepsilon]}{\delta \varepsilon_{i}(x)}\right|_{\varepsilon=0},
$$

where the index $i=1, \cdots, N$ labels the $N$ composite operators which we wish to consider. Clearly, the same information is also encoded in the source dependent EAA. One then defines the composite operator $\left[O_{i}\right]$ by taking a functional derivative with respect to the associated source:

$$
\left.\left[O_{k}(x)\right]_{i} \equiv \frac{\delta}{\delta \varepsilon_{i}(x)} \Gamma_{k}[\varphi, \varepsilon]\right|_{\varepsilon=0}=\left\langle O_{i}(x)\right\rangle_{J[\varphi]} .
$$

The so defined composite operator satisfies its own flow equation, which is given by [60-63]:

$$
\partial_{t}\left(\varepsilon \cdot\left[O_{k}\right]\right)=-\frac{1}{2} \operatorname{Tr}\left[\left(\Gamma_{k}^{(2)}+\mathcal{R}_{k}\right)^{-1}\left(\varepsilon \cdot\left[O_{k}\right]^{(2)}\right)\left(\Gamma_{k}^{(2)}+\mathcal{R}_{k}\right)^{-1} \partial_{t} \mathcal{R}_{k}\right],
$$

where $\left[O_{k}\right]^{(2)}$ is the Hessian of the composite operator.

To solve Equation (11) one must implement some approximations. A possible strategy is to project the composite operator $\left[O_{k}\right]$ on the truncated set of operators $\left\{O_{1}, \cdots, O_{N}\right\}$ :

$$
\left[O_{k}(x)\right]=\sum_{j=1}^{N} Z_{i j}(k) O_{j}(x)
$$

In practical calculations we will limit ourselves to non-mixing ansätze, where the composite operator is parametrized by

$$
\left[O_{k}(x)\right]=Z(k) O(x),
$$

where $Z(k)$ encodes the RG evolution of the operator. The scaling dimension associated with the ansatz (13) can be used to estimate the scaling dimension $\Delta_{O}$ of the operator $O(x)$ via

$$
\Delta_{O}=d_{O}+\gamma_{O},
$$

where $d_{O}$ is the classical mass dimension of the operator $O(x)$ and $\gamma_{O} \equiv Z_{O}^{-1} \partial_{t} Z_{O}{ }^{3}$

3 Similar non-mixing ansätze have been already employed in the literature $[52,64,65]$. 
We are now in the position of discussing the application of this framework to investigate the scaling properties of geometric operators. Let us consider the case of the volume of an n-dimensional hypersurface:

$$
V_{\sigma_{n}}=\int_{U} d^{n} u \sqrt{\operatorname{det} g(u)}
$$

To introduce $V_{\sigma_{n}}$ in our formalism we add to the microscopic action the following source dependent term:

$$
\int_{U} d^{n} u \varepsilon(u) \sqrt{\operatorname{det} g(u)} .
$$

Using a non-mixing ansatz for the source dependent EAA, we add the following term to the Einstein-Hilber truncation for $\Gamma_{k}[g]$ :

$$
\int_{U} d^{n} u \varepsilon(u) Z_{\sigma_{n}}(k) \sqrt{\operatorname{det} g(u)} .
$$

In the case of a non-mixing ansatz for $V_{\sigma_{n}}$ we can also simply set $\varepsilon(u)$ to a constant and keep it as a bookkeeping parameter. Inserting the ansatz (14) into Equation (11) it is straightforward to rewrite the flow equation for composite operators as

$$
\gamma_{\sigma_{n}} V_{\sigma_{n}}=-\frac{1}{2} \operatorname{Tr}\left[\left(\Gamma_{k}^{(2)}+\mathcal{R}_{k}\right)^{-1}\left(V_{\sigma_{n}}^{(2)}\right)\left(\Gamma_{k}^{(2)}+\mathcal{R}_{k}\right)^{-1} \partial_{t} \mathcal{R}_{k}\right],
$$

which is expressed directly in terms of the anomalous dimension. In this approximation, the anomalous dimension associated with $V_{\sigma_{n}}$ is given by $\gamma_{\sigma_{n}} \equiv Z_{\sigma_{n}}^{-1} \partial_{t} Z_{\sigma_{n}}$. Since the classical mass dimension of $V_{\sigma_{n}}$ is $d_{\sigma_{n}}=-n$, we have that the full scaling dimension reads $\Delta_{\sigma_{n}}=-n+\gamma_{\sigma_{n}}$, which implies an effective reduction of the scaling dimension if $\gamma_{\sigma_{n}}>0$.

\section{Scaling Properties of Geometric Operators}

\subsection{Volume of Hypersurfaces}

In this section, we study the anomalous scaling of hypersurfaces in the Einstein-Hilbert truncation by varying the approximations employed and the field content considered.

The Einstein-Hilbert truncation (9) and the associated running given by the flow Equation (8) has been studied several times in the literature, see $[66,67]$ for an overview. Depending on certain choices, such as cutoff profile and gauge-fixing, one obtains different values of the Newton's constant and the cosmological constant. The values of the couplings at the fixed point are not physical quantities and typically vary by changing the aforementioned choices. However, such dependences should cancel in actual observable quantities, such as the critical exponents. Of course, when approximations are made, one only expects that the calculation of physical quantities is at best only weakly influenced by these choices, such as that of the cutoff profile.

The anomalous dimension, being related to physical quantities, is also expected to be independent of unphysical choices. In this section we evaluate the anomalous dimension in various settings. Given that the truncations for the gravitational EAA and the composite operators are very crude, the framework employed in this work is purely exploratory. Despite this fact we expect to be able to grasp the qualitative feature encoded in the anomalous dimensions. As we shall see, the main feature of all the anomalous dimensions computed in this work is that their value is always positive, thus hinting to an effective dimensional reduction of the scaling dimension of the geometric operators. 
Given the Einstein-Hilbert truncation and the non-mixing ansatz for the volume of a hypersurface, one can explicitly compute the RHS of Equation (15). The details of the calculation can be found in [64]. The resulting flow equation is given by [64]:

$$
\begin{aligned}
\gamma_{\sigma_{n}}\left(\lambda_{k}, g_{k}\right) \equiv \gamma_{\sigma_{n}}(k)= & 2\left(\frac{1}{4 \pi}\right)^{\frac{d}{2}-1}\left[\frac{(d+1) n^{2}-2 n}{d}+\frac{4 n-2 n^{2}}{2 d-d^{2}}\right] \\
& \times g_{k}\left[\Phi_{d / 2}^{2}\left(-2 \lambda_{k}\right)-\frac{1}{2} \eta_{N}\left(g_{k}, \lambda_{k}\right) \tilde{\Phi}_{d / 2}^{2}\left(-2 \lambda_{k}\right)\right],
\end{aligned}
$$

where $g_{k} \equiv G_{k} k^{d-2}$ and $\lambda_{k} \equiv \Lambda_{k} k^{-2}$ are the dimensionless Newton's and cosmological constants and we have introduced the threshold functions [2]:

$$
\begin{aligned}
\Phi_{n}^{p}(w) & \equiv \frac{1}{\Gamma(n)} \int_{0}^{\infty} d z z^{n-1} \frac{R^{(0)}(z)-z R^{(0) \prime}(z)}{\left[z+R^{(0)}(z)+w\right]^{p}} \text { and } \\
\tilde{\Phi}_{n}^{p}(w) & \equiv \frac{1}{\Gamma(n)} \int_{0}^{\infty} d z z^{n-1} \frac{R^{(0)}(z)}{\left[z+R^{(0)}(z)+w\right]^{p}} .
\end{aligned}
$$

The RHS of Equation (16) can be evaluated in several ways. In particular, in Section 4.1.1 we compute the anomalous dimensions in the one-loop approximation, also considering the possible addition of matter fields. In Section 4.1.2 we compute the anomalous dimensions in the full-fledged single metric truncation and report explicit values for the cases $d=3$ and $d=4$. Finally, we compute the value of the anomalous dimensions in a bimetric setting by taking into account the graviton anomalous dimension in Section 4.1.3.

Explicit values are obtained using the optimized cutoff $\mathcal{R}_{k}=\left(\Delta-k^{2}\right) \theta\left(\Delta-k^{2}\right)$ [68] and the exponential cutoff $\mathcal{R}_{k}=s \Delta /\left(e^{s \Delta / k^{2}}-1\right)$. By computing the anomalous dimensions via different cutoff profiles one can estimate the uncertainty due to the cutoff choice. This gives an approximate lower bound of the error associated with the obtained value for $\gamma_{\sigma_{n}}$. A further estimate of the error can be found by comparing the results obtained by systematically enlarging the truncation for the EAA and/or the composite operators. We emphasize that in a non-perturbative framework such as the FRG, such a procedure may in principle change rather drastically the numerical values of $\gamma_{\sigma_{n}}$ with respect to those obtained in the following, which are based on a crude approximation scheme and vary over a significant range when comparing the results of Sections 4.1.1, 4.1.2, and 4.1.3. For this reason, our work has an exploratory nature rather than a quantitative one.

\subsubsection{Scaling of $V_{\sigma_{n}}$ at One-Loop}

In the one-loop approximation the RHS of Equation (16) is computed as follows. First one sets the anomalous dimension $\eta_{N}$ to zero. Moreover, one performs a coupling expansion to the first non-trivial order. In practice this boils down to evaluate only the threshold functional $\Phi$ at a vanishing value of the cosmological constant.

We now evaluate the anomalous dimensions $\gamma_{\sigma_{n}}$ via this approximation in various settings.

Pure gravity in $d=4$. In Table 1 we report the values of the one-loop fixed-point anomalous dimensions $\gamma_{\sigma_{n}}$. The uncertainty due to the cutoff choice is roughly the $3 \%$ of the anomalous dimension.

Table 1. Fixed-point values of $\gamma_{\sigma_{n}}$ for $d=4$. The first and second column report the results obtained via the optimized and the exponential cutoff, respectively.

\begin{tabular}{ccc}
\hline & $\gamma_{\sigma_{n}}^{\text {opt,1L}}\left(g_{*}^{\text {opt,1L }}\right)$ & $\gamma_{\sigma_{n}}^{\text {exp,1L }}\left(g_{*}^{\mathbf{e x p}, 1 \mathrm{~L}}\right)$ \\
\hline$n=1$ & 0.0682 & 0.0671 \\
$n=2$ & 0.5455 & 0.5368 \\
$n=3$ & 1.4318 & 1.4091 \\
\hline
\end{tabular}


Pure gravity in $d=3$. The one-loop expression for $\gamma_{\sigma_{n}}$ is particularly simple due to a cancellation between the dependence of both $g_{*}$ and the RHS of (16) on the threshold functions. One finds

$$
\gamma_{\sigma_{n}}=\frac{n(n-1)}{5} .
$$

Gravity in $d=4$ with matter fields. We now report the one-loop fixed-point value of the anomalous dimension $\gamma_{\sigma_{n}}$ when matter fields are added. Following the nomenclature of [16] we employ type II cutoffs for all matter fields. Using the optimized cutoff, one finds

$$
\gamma_{\sigma_{n}}=\frac{12 \pi}{22+4 n_{V}-n_{S}-2 n_{D}} \cdot \frac{n(3 n-2)}{8 \pi} .
$$

The anomalous dimension (19) is proportional to the fixed-point Newton's constant. Please note that for certain choices of matter content, i.e., of $\left(n_{V}, n_{S}, n_{D}\right)$, the fixed-point Newton's constant can be even negative, implying that $\gamma_{\sigma_{n}}$ is negative in this case, too. However, one requires that the AS fixed point has a positive Newton's constant. This requirement gives a non-trivial constraint on the matter content compatible with the AS scenario, we refer to [67] for a more detailed discussion of these issues. In the present case a positive value of the fixed-point Newton's constant implies a positive value of $\gamma_{\sigma_{n}}$.

\subsubsection{Scaling of $V_{\sigma_{n}}$ in the Full-Fledge Einstein-Hilbert Truncation}

Let us go back to the RHS of Equation (16). We now consider that $\eta_{N}=\partial_{t} G_{k} / G_{k}=2-d$ at the fixed point. We report below the results for the pure gravity case in $d=3$ and $d=4$.

Pure gravity in $d=4$. In Table 2 we report the fixed-point values of the anomalous dimensions of hypersurfaces. We checked that by varying the $s$ parameter in the exponential cutoff that the anomalous dimensions vary at most by $3 \%$ with respect to their values.

Table 2. Fixed-point values of $\gamma_{\sigma_{n}}$ in $d=4$ for the full-fledged single metric Einstein-Hilbert truncation.

\begin{tabular}{ccc}
\hline & $\gamma_{\sigma_{n}}^{\text {opt }}\left(\lambda_{*}^{\text {opt }}, g_{*}^{\text {opt }}\right)$ & $\gamma_{\sigma_{n}}^{\text {exp }}\left(\lambda_{*}^{\text {exp }}, g_{*}^{\text {exp }}\right)$ \\
\hline$n=1$ & 0.0997 & 0.1006 \\
$n=2$ & 0.7973 & 0.8044 \\
$n=3$ & 2.0930 & 2.1116 \\
\hline
\end{tabular}

Pure gravity in $d=3$. The values of the anomalous dimension are reported in Table 3 . (The uncertainty due to the choice of the cutoff profile is estimated to be of the order of $6 \%$.)

Table 3. Fixed-point values of $\gamma_{\sigma_{n}}$ in $d=3$ in the full-fledged Einstein-Hilbert truncation.

\begin{tabular}{ccc}
\hline & $\gamma_{\sigma_{n}}^{\text {opt }}\left(\lambda_{*}^{\text {opt }}, g_{*}^{\text {opt }}\right)$ & $\gamma_{\sigma_{n}}^{\exp }\left(\lambda_{*}^{\text {exp }}, g_{*}^{\text {exp }}\right)$ \\
\hline$n=1$ & 0 & 0 \\
$n=2$ & 0.5303 & 0.5692 \\
$n=3$ & 1.5908 & 1.7076 \\
\hline
\end{tabular}

We note that in this approximation the anomalous dimensions are larger than the one-loop ones of Section 4.1.1.

\subsubsection{Scaling of $V_{\sigma_{n}}$ in the Improved Einstein-Hilbert Truncation}

We now consider the Einstein-Hilbert truncation (9) and redefine the fluctuating metric via $h_{\mu v} \rightarrow \sqrt{32 \pi G_{k}} Z_{k}^{1 / 2} h_{\mu v}$, where we have introduced a wave function renormalization constant $Z_{k}$ for the graviton which is independent of the Newton's constant. Employing this definition allows one to 
make a first distinction between the zero level couplings, i.e., the couplings associated with $\Gamma_{k}[\bar{g}, 0]$, and the level two couplings, which are related to $\frac{\delta^{2}}{\delta h \delta h} \Gamma_{k}[\bar{g}, 0]$.

The difference with respect to the ansatz of section 4.1.2 is that now the anomalous dimension $\eta_{N}$ is not determined by the Newton's constant but it is calculated independently by computing $\eta_{N}=-Z_{k}^{-1} \partial_{t} Z_{k}$. The fixed-point value of the graviton anomalous dimension has already been computed via slightly different procedures in the literature, (see e.g., [67] for an overview). In Table 4 we report the values of the anomalous dimensions $\gamma_{\sigma_{n}}$ found in this approximation by using the fixed-point coupling constants and anomalous dimension given in [67].

Table 4. Fixed-point values of $\gamma_{\sigma_{n}}$ for $d=4$ using the graviton anomalous dimension computed via $\eta_{N}=-Z_{k}^{-1} \partial_{t} Z_{k}$

\begin{tabular}{ccc}
\hline$\gamma_{\sigma_{1}}^{\text {opt }}\left(g_{*}^{\text {opt }}\right)$ & $\gamma_{\sigma_{2}}^{\text {opt }}\left(g_{*}^{\text {opt }}\right)$ & $\gamma_{\sigma_{3}}^{\text {opt }}\left(g_{*}^{\text {opt }}\right)$ \\
\hline 0.0474 & 0.3794 & 0.9959 \\
\hline
\end{tabular}

From the values in Table 4 we can see that the anomalous dimensions $\gamma_{\sigma_{n}}$ at the fixed point are always positive with a smaller value compared to those of Section 4.1.2.

Finally, it would be interesting to extend the present results to other kinds of gravitational theories, such as those with an independent connection, that have shown compatibility with the AS program [69-78].

\subsection{The Geodesic Length and Its Uses}

\subsubsection{The Geodesic Length}

In this section, we study the scaling property of the geodesic length $\ell_{g}$, which is defined by

$$
\ell_{g} \equiv \int_{0}^{1} d \tau \sqrt{g_{\mu v}\left(x_{g}(\tau)\right) \dot{x}_{g}^{\mu}(\tau) \dot{x}_{g}^{\nu}(\tau)}
$$

where the trajectory $x_{g}^{\mu}(\tau)$ satisfies the following equation

$$
\ddot{x}_{g}^{\mu}(\tau)+\Gamma_{\alpha}^{\mu}{ }_{\beta}\left(x_{g}(\tau)\right) \dot{x}_{g}^{\alpha}(\tau) \dot{x}_{g}^{\beta}(\tau)=0 .
$$

We emphasize that the so defined trajectory $x_{g}^{\mu}(\tau)$ has an implicit, i.e., functional, dependence on the metric $g_{\mu v}$. Because of this, the length $\ell_{g}$ has further new "graviton vertices" with respect to the case of the length $\ell$ of a prescribed trajectory, which we have considered in Section 4.1.

It is important to note that to fully specify the trajectory $x_{g}^{\mu}(\tau)$ it is necessary to state which boundary/initial conditions are associated to Equation (21). Let us describe three sensible options.

(i) Boundary value problem. One requires that the solution of Equation (21) satisfies

$$
\left\{\begin{array}{l}
x^{\mu}(0)=x_{0}^{\mu} \\
x^{\mu}(1)=x_{1}^{\mu} .
\end{array}\right.
$$

(ii) Initial value problem. One requires that the solution of Equation (21) satisfies

$$
\left\{\begin{array}{l}
x^{\mu}(0)=x_{0}^{\mu} \\
\dot{x}^{\mu}(0)=v_{0}^{\mu} .
\end{array}\right.
$$


(iii) Normalized initial value problem at fixed geodesic length. One requires that the solution of Equation (21) satisfies

$$
\left\{\begin{array}{l}
x^{\mu}(0)=x_{0}^{\mu} \\
\frac{\dot{x}^{\mu}(0)}{\left\|\dot{x}_{0}\right\|}=\xi_{0}^{\mu} \\
\ell_{g}\left(x_{0}, x_{g}(1)\right)=r .
\end{array}\right.
$$

In the following we will limit ourselves to discuss the case of the boundary conditions (22) and will comment on the other cases at the end of the section. It must be stressed that boundary conditions are taken to be independent from the metric, i.e., there is no implicit (functional) metric dependence in $x_{0}^{\mu}$ and $x_{1}^{\mu}$.

The crucial feature of the flow Equation (11) is the presence of the Hessian of the composite operator on the RHS. In full analogy with Equation (15), one must solve the following equation

$$
\gamma_{\ell_{g}} \ell_{g}=-\frac{1}{2} \operatorname{Tr}\left[\left(\Gamma_{k}^{(2)}+\mathcal{R}_{k}\right)^{-1}\left(\ell_{g}^{(2)}\right)\left(\Gamma_{k}^{(2)}+\mathcal{R}_{k}\right)^{-1} \partial_{t} \mathcal{R}_{k}\right]
$$

In the present case, thus, we must compute the Hessian of the geodesic length. We now summarize the strategy adopted in [64] and refer the reader to [64] for further details.

To compute the Hessian of $\ell_{g}$ one must know the dependence of $x_{g}^{\mu}(\tau)$ on the fluctuation $h_{\mu v}$. We work on a flat background, i.e., $\bar{g}_{\mu v}=\delta_{\mu v}$, and expand the geodesic trajectory as follows

$$
x_{g}^{\mu}(\tau)=x_{0}^{\mu}\left[h^{0}\right](\tau)+x_{1}^{\mu}[h](\tau)+\frac{1}{2} x_{2}^{\mu}\left[h^{2}\right](\tau)+\cdots,
$$

where $x_{1}^{\mu}[h]$ is linear in $h_{\mu v}, x_{2}^{\mu}\left[h^{2}\right]$ is quadratic in $h_{\mu v}$ and so on. The explicit expression for $x_{i}^{\mu}$ can be found as follows. First one expands the connection in the geodesic equation up to the desired order, in our case

$$
\ddot{x}_{g}^{\mu}(\tau)+\left(\left.\Gamma_{\alpha}^{\mu}\right|_{\bar{g}=\delta}+\left.\delta \Gamma_{\alpha}^{\mu}\right|_{\bar{g}=\delta}+\left.\frac{1}{2} \delta^{2} \Gamma_{\alpha}^{\mu}\right|_{\bar{g}=\delta}\right) \dot{x}_{g}^{\alpha}(\tau) \dot{x}_{g}^{\beta}(\tau)=0 \text {. }
$$

One then inserts the expansion (26) into (27) and solves the equation order by order. This procedure is straightforward. The only caveat is that one must ensure the boundary conditions (22) are satisfied at each order. For example, the solution of the zeroth order term is trivial, one has

$$
\begin{aligned}
& \ddot{x}_{0}^{\mu}(\tau)=0 \\
& x_{0}^{\mu}(\tau)=x_{0}^{\mu}+\xi_{0}^{\mu} \tau,
\end{aligned}
$$

with $\xi_{0}^{\mu}=x_{1}^{\mu}-x_{0}^{\mu}$.

To extract the anomalous dimension from Equation (25) we project onto flat space, i.e., we set $\bar{g}_{\mu \nu}=$ $\delta_{\mu v}$ and $h_{\mu \nu}=0$. The LHS of (25) is thus simply proportional to $\ell_{g=\delta}=\sqrt{\delta_{\mu \nu} \xi_{0}^{\mu} \xi_{0}^{v}}$. The anomalous dimension is then determined by extracting the terms proportional to $\xi_{0}$ in the RHS of (25).

An explicit computation shows that among all the terms on the RHS, those proportional to $\xi_{0}$ are the ones that come from the explicit dependence of the metric in (20). No contributions actually come from the graviton vertices of $x_{g}^{\mu}(\tau)$. The latter induce mixing with other operators which are neglected in our simple non-mixing ansatz. It follows that the relevant contributions are the same as the ones relevant for a generic length $\ell$. In turn, all of this implies that in our approximation the anomalous dimension of $\ell_{g}$ is the same as that of $\ell=V_{\sigma_{1}}$. Therefore, for the geodesic length with boundary conditions one has $\gamma_{\ell_{g}}=\gamma_{\sigma_{1}}$ and that the results found in Sections 4.1.1-4.1.3 apply to $\ell_{g}$ as well.

Finally, let us come back to the choice of initial/boundary condition. Following the arguments in [64], it is possible to check that also in the case of the initial value problem defined by (23) one has $\gamma_{\ell_{g}}=\gamma_{\sigma_{1}}$. However, an explicit calculation shows that the Hessians of $\ell_{g}$ associated with the 
boundary and the initial value problems are different. Therefore, one expects their scaling properties to be different in general and this difference to be grasped by employing more refined mixing ansätze for the composite operators.

Finally, let us consider the normalized initial value problem defined by (24). This case is very different from the previous ones. In particular, due to the third equation in (24), the trajectory $x_{g}^{\mu}(\tau)$ is such that $\ell_{g}=r$, where $r$ is a given number which is independent of the metric. It follows that no anomalous dimension is associated with $\ell_{g}$ since the latter is taken to be a given number by definition. Such a definition has been used in the literature to define another kind of geodesic fixed correlation length $[79,80]$, which should not be confused with the correlation defined by (5) which employs a boundary value problem. This last example clarifies that to study the scaling properties of the geodesic length one must carefully define how the geodesic trajectory is actually defined and in particular which type of boundary or initial conditions one wants to employ.

\subsubsection{The Geodesic Ball and the Geodesic Sphere}

We now consider further geometric operators, which can be defined thanks to the geodesic distance.

Let us start by considering the volume of a geodesic ball. We locate the center of the ball at a point $x_{0}^{\mu}$, which we take as a given condition independent of the metric. The volume of the geodesic ball is defined by

$$
V_{\text {ball }}(r) \equiv \int_{B} d^{d} x \sqrt{g}
$$

where the domain of integration is

$$
B \equiv\left\{x: \lg \left(x, x_{0}\right) \leq r\right\} .
$$

In (28) $\ell_{g}\left(x, x_{0}\right)$ denotes the geodesic distance between $x_{0}$ and $x$ while $r$ is a given number, which is independent of the metric. ${ }^{4}$

The volume of the geodesic ball can be expressed as an expansion in $r$ around $r=0$ (see e.g., [81]):

$$
V_{\text {ball }}(r)=\frac{2 \Gamma\left(\frac{1}{2}\right)^{d}}{d \Gamma\left(\frac{d}{2}\right)}\left\{r^{d}-\frac{R\left(x_{0}\right)}{6(d+2)} r^{d+2}+\cdots\right\} .
$$

We are particularly interested in the small $r$ limit, since the latter is related to the Hausdorff dimension $d_{\mathrm{H}}$ via the following scaling relation [59]

$$
\lim _{r \rightarrow 0}\left\langle V_{\text {ball }}(r)\right\rangle \sim r^{d_{\mathrm{H}}} .
$$

Given the expansion (29) one may suspect that only the first term is relevant. If we assume that this is the case one immediately obtains that $d_{\mathrm{H}}=d$ since $\left\langle r^{d}\right\rangle=r^{d}$ due to the fact that $r$ is a given number independent of the metric. In this case, thus, one finds that the Hausdorff dimension corresponds to the topological dimension.

To inspect if the other terms in (29) are negligible we estimate the contribution of the second term. To do so we employ a sort of mean field analysis where $\left\langle O\left(g_{\mu \nu}\right)\right\rangle \approx O\left(g_{k, \mu v}^{s c}\right)$, with $g_{k, \mu \nu}^{s c}$ being the so-called self-consistent metric [82]. In the UV regime one then has

$$
\left\langle R\left(x_{0}\right) r^{d+2}\right\rangle \sim r^{d}(k r)^{2},
$$


which blows up like $k^{2}$ for $k \rightarrow \infty$ and vanishes like $r^{d+2}$ in the limit $r \rightarrow 0$. It follows that the higher curvature terms in (29) are negligible if $k r \ll 1$. The condition $k r \ll 1$ actually corresponds to require that the ball radius is inside the range of lengths that have been integrated out by the RG flow, i.e., $0 \ll r \ll k^{-1}$. This is a physical requirement since only in this way the ball is affected by all the relevant modes. This argument thus confirms that the leading contribution to $\left\langle V_{\text {ball }}\right\rangle$ is proportional to $r^{d}$ so that $d_{\mathrm{H}}=d$. The relation $d_{\mathrm{H}}=d$ was already known in the AS literature via a different argument, we refer the reader to [59] for further details.

It is straightforward to extend these considerations to the volume of a geodesic sphere, which is defined as the set of points that satisfies $\ell_{g}\left(x, x_{0}\right)=r$. In this case, one has

$$
V_{\text {sphere }}(r)=\frac{2 \Gamma\left(\frac{1}{2}\right)^{d}}{\Gamma\left(\frac{d}{2}\right)}\left\{r^{d-1}-\frac{R\left(x_{0}\right)}{6 d} r^{d+1}+\cdots\right\} .
$$

In the small $r$ limit one then obtains $\left\langle V_{\text {sphere }}\right\rangle \sim r^{d-1}$.

\section{Summary}

In this paper, we reviewed the role of geometric operators in the AS scenario for quantum gravity and described how one can study the scaling properties of such operators in the framework of the functional renormalization group. We have also reported on the study of the anomalous dimensions of geometric operators under certain approximations that have not been considered in the literature before.

In particular, in Section 2 we introduced the volume of hypersurfaces and the geodesic length and discussed their possible use in the AS scenario for quantum gravity. In Section 3 we introduced the Einstein-Hilbert truncation for the gravitational EAA and discussed how to study geometric operators as composite operators in the FRG framework. In Section 4 we studied the scaling properties of the composite operators in different settings. It turns out that under all the approximations considered the anomalous dimensions are positive implying an effective reduction of the scaling properties of the geometric operators. Finally, the scaling properties of a geodesic ball have been investigated and it has been argued that the Hausdorff dimension corresponds to the topological one in the AS scenario.

We emphasize, however, that the nature of the present study is exploratory. To reach a quantitative determination of the scaling properties one should extend our study by considering increasingly complicated truncations for the EAA and introducing mixing ansätze for the composite operators. It follows that more work is needed in this direction to be able to possibly make comparisons with other approaches to quantum gravity. Indeed, the study of geometric operators paves the way to the study of observables such as the correlation function defined in (5), which one may hope to compare with results from other approaches, such as Causal Dynamical Triangulations [83].

Author Contributions: All the authors contributed equally to this work.

Funding: The research of M.B. was funded by Deutsche Forschungsgemeinschaft (DFG) under the Grant No. RE 793/8-1.

Acknowledgments: The authors are grateful to Martin Reuter for many inspiring and enlightening discussions on this and many other topics. The authors are also grateful to the organizers and the participants of the workshop "Quantum Fields-From Fundamental Concepts to Phenomenological Questions" for many interesting discussions and the feedback on the present work.

Conflicts of Interest: The authors declare no conflict of interest.

\section{References}

1. Weinberg, S. General Relativity, an Einstein Centenary Survey; Hawking, S.W., Israel, W., Eds.; Cambridge University Press: New York, NY, USA, 1980; p. 790.

2. Reuter, M. Nonperturbative Evolution Equation for Quantum Gravity. Phys. Rev. D 1998, 57, 971. [CrossRef] 
3. Wetterich, C. Exact evolution equation for the effective potential. Phys. Lett. B 1993, 301, 90-94. [CrossRef]

4. Manrique, E.; Reuter, M. Bare Action and Regularized Functional Integral of Asymptotically Safe Quantum Gravity. Phys. Rev. D 2009, 79, 025008. [CrossRef]

5. Bonanno, A.; Reuter, M. Renormalization group improved black hole space-times. Phys. Rev. D 2000, 62, 043008. [CrossRef]

6. Bonanno, A.; Reuter, M. Cosmology of the Planck era from a renormalization group for quantum gravity. Phys. Rev. D 2002, 65, 043508. [CrossRef]

7. Bonanno, A.; Reuter, M. Entropy signature of the running cosmological constant. J. Cosmol. Astropart. Phys. 2007, 2007, 024. [CrossRef]

8. Koch, B.; Saueressig, F. Structural aspects of asymptotically safe black holes. Class. Quant. Grav. 2014, 31, 015006. [CrossRef]

9. Bonanno, A.; Saueressig, F. Asymptotically safe cosmology-A status report. Comptes Rendus Phys. 2017, 18, 254-264. [CrossRef]

10. Bonanno, A.; Platania, A.; Saueressig, F. Cosmological bounds on the field content of asymptotically safe gravity-matter models. Phys. Lett. B 2018, 784, 229-236. [CrossRef]

11. Ellwanger, U. FLow equations for $\mathrm{N}$ point functions and bound states. Z. Phys. C 1994, 62, 503-510. [CrossRef]

12. Morris, T.R. The Exact renormalization group and approximate solutions. Int. J. Mod. Phys. A 1994, 9, 2411-2419. [CrossRef]

13. Lauscher, O.; Reuter, M. Flow equation of quantum Einstein gravity in a higher derivative truncation. Phys. Rev. D 2002, 66, 025026. [CrossRef]

14. Reuter, M.; Saueressig, F. A Class of nonlocal truncations in quantum Einstein gravity and its renormalization group behavior. Phys. Rev. D 2002, 66, 125001. [CrossRef]

15. Codello, A.; Percacci, R.; Rahmede, C. Ultraviolet properties of f(R)-gravity. Int. J. Mod. Phys. A 2008, 23, 143-150. [CrossRef]

16. Codello, A.; Percacci, R.; Rahmede, C. Investigating the Ultraviolet Properties of Gravity with a Wilsonian Renormalization Group Equation. Ann. Phys. 2009, 324, 414-469. [CrossRef]

17. Benedetti, D.; Machado, P.; Saueressig, F. Asymptotic safety in higher-derivative gravity. Mod. Phys. Lett. A 2009, 24, 2233-2241. [CrossRef]

18. Benedetti, D.; Groh, K.; Machado, P.; Saueressig, F. The Universal RG Machine. J. High Energy Phys. 2011, 2011, 079. [CrossRef]

19. Ohta, N.; Percacci, R. Higher Derivative Gravity and Asymptotic Safety in Diverse Dimensions. Class. Quant. Grav. 2014, 31, 015024. [CrossRef]

20. Benedetti, D. On the number of relevant operators in asymptotically safe gravity. Europhys. Lett. 2013, 102, 20007. [CrossRef]

21. Gies, H.; Knorr, B.; Lippoldt, S.; Saueressig, F. Gravitational Two-Loop Counterterm Is Asymptotically Safe. Phys. Rev. Lett. 2016, 116, 211302. [CrossRef] [PubMed]

22. Falls, K.; Litim, D.; Nikolakopoulos, K.; Rahmede, C. Further evidence for asymptotic safety of quantum gravity. Phys. Rev. D 2016, 93, 104022. [CrossRef]

23. Ohta, N.; Percacci, R.; Vacca, G. Flow equation for $f(R)$ gravity and some of its exact solutions. Phys. Rev. $D$ 2015, 92, 061501. [CrossRef]

24. Falls, K.; Litim, D.; Nikolakopoulos, K.; Rahmede, C. On de Sitter solutions in asymptotically safe $f(R)$ theories. arXiv 2016, arXiv:1607.04962.

25. Falls, K.; Ohta, N. Renormalization Group Equation for $f(R)$ gravity on hyperbolic spaces. Phys. Rev. D 2016, 94, 084005. [CrossRef]

26. Reuter, M.; Weyer, H. Conformal sector of Quantum Einstein Gravity in the local potential approximation: Non-Gaussian fixed point and a phase of unbroken diffeomorphism invariance. Phys. Rev. D 2009, 80, 025001. [CrossRef]

27. Benedetti, D.; Caravelli, F. The Local potential approximation in quantum gravity. J. High Energy Phys. 2012, 2012, 017. [CrossRef] 
28. Demmel, M.; Saueressig, F.; Zanusso, O. Fixed-Functionals of three-dimensional Quantum Einstein Gravity. J. High Energy Phys. 2012, 2012, 131. [CrossRef]

29. Dietz, J.; Morris, T. Asymptotic safety in the $\mathrm{f}(\mathrm{R})$ approximation. J. High Energy Phys. 2013, $2013,108$. [CrossRef]

30. Bridle, I.H.; Dietz, J.A.; Morris, T.R. The local potential approximation in the background field formalism. J. High Energy Phys. 2014, 2014, 093. [CrossRef]

31. Dietz, J.A.; Morris, T.R. Redundant operators in the exact renormalisation group and in the $f(R)$ approximation to asymptotic safety. J. High Energy Phys. 2013, 2013, 064. [CrossRef]

32. Demmel, M.; Saueressig, F.; Zanusso, O. RG flows of Quantum Einstein Gravity on maximally symmetric spaces. J. High Energy Phys. 2014, 2014, 026. [CrossRef]

33. Demmel, M.; Saueressig, F.; Zanusso, O. RG flows of Quantum Einstein Gravity in the linear-geometric approximation. Ann. Phys. 2015, 359, 141. [CrossRef]

34. Demmel, M.; Saueressig, F.; Zanusso, O. A proper fixed functional for four-dimensional Quantum Einstein Gravity. J. High Energy Phys. 2015, 2015, 113. [CrossRef]

35. Ohta, N.; Percacci, R.; Vacca, G.P. Renormalization Group Equation and scaling solutions for $f(R)$ gravity in exponential parametrization. Eur. Phys. J. C 2016, 76, 46. [CrossRef]

36. Labus, P.; Morris, T.R.; Slade, Z.H. Background independence in a background dependent renormalization group. Phys. Rev. D 2016, 94, 024007. [CrossRef]

37. Dietz, J.A.; Morris, T.R.; Slade, Z.H. Fixed point structure of the conformal factor field in quantum gravity. Phys. Rev. D 2016, 94, 124014. [CrossRef]

38. Knorr, B. Infinite order quantum-gravitational correlations. arXiv 2017, arXiv:1710.07055.

39. Falls, K.G.; King, C.S.; Litim, D.F.; Nikolakopoulos, K.; Rahmede, C. Asymptotic safety of quantum gravity beyond Ricci scalars. arXiv 2017, arXiv:1801.00162.

40. Alkofer, N.; Saueressig, F. Asymptotically safe $f(R)$-gravity coupled to matter I: the polynomial case. arXiv 2018, arXiv:1802.00498.

41. Manrique, E.; Reuter, M. Bimetric Truncations for Quantum Einstein Gravity and Asymptotic Safety. Ann. Phys. 2010, 325, 785-815. [CrossRef]

42. Manrique, E.; Reuter, M.; Saueressig, F. Matter Induced Bimetric Actions for Gravity. Ann. Phys. 2011, 326, 440-462. [CrossRef]

43. Manrique, E.; Reuter, M.; Saueressig, F. Bimetric Renormalization Group Flows in Quantum Einstein Gravity. Ann. Phys. 2011, 326, 463-485. [CrossRef]

44. Becker, D.; Reuter, M. En route to Background Independence: Broken split-symmetry, and how to restore it with bi-metric average actions. Ann. Phys. 2014, 350, 225-301. [CrossRef]

45. Christiansen, N.; Litim, D.F.; Pawlowski, J.M.; Rodigast, A. Fixed points and infrared completion of quantum gravity. Phys. Lett. B 2014, 728, 114-117. [CrossRef]

46. Codello, A.; D'Odorico, G.; Pagani, C. Consistent closure of renormalization group flow equations in quantum gravity. Phys. Rev. D 2014, 89, 081701. [CrossRef]

47. Christiansen, N.; Knorr, B.; Pawlowski, J.M.; Rodigast, A. Global Flows in Quantum Gravity. Phys. Rev. D 2016, 93, 044036. [CrossRef]

48. Christiansen, N.; Knorr, B.; Meibohm, J.; Pawlowski, J.M.; Reichert, M. Local Quantum Gravity. Phys. Rev. D 2015, 92, 121501. [CrossRef]

49. Knorr, B.; Lippoldt, S. Correlation functions on a curved background. Phys. Rev. D 2017, 96, 065020. [CrossRef]

50. Christiansen, N.; Falls, K.; Pawlowski, J.M.; Reichert, M. Curvature dependence of quantum gravity. Phys. Rev. D 2018, 97, 046007. [CrossRef]

51. Eichhorn, A.; Labus, P.; Pawlowski, J.M.; Reichert, M. Effective universality in quantum gravity. arXiv 2018, arXiv:1804.00012.

52. Pagani, C.; Reuter, M. Composite Operators in Asymptotic Safety. Phys. Rev. D 2017, 95, 066002. [CrossRef] 
53. Pagani, C.; Reuter, M. Finite Entanglement Entropy in Asymptotically Safe Quantum Gravity. J. High Energy Phys. 2018, 2018, 039. [CrossRef]

54. Carlip, S. Dimension and Dimensional Reduction in Quantum Gravity. Class. Quant. Grav. 2017, $34,193001$. [CrossRef]

55. Ambjørn, J.; Durhuus, B.; Jonsson, T. Quantum Geometry: A Statistical Field Theory Approach; Cambridge University Press: Cambridge, UK, 1997.

56. Hamber, H.W. Quantum Gravitation: The Feynman Path Integral Approach; Springer: Berlin, Germany, 2009.

57. Codello, A.; D'Odorico, G. Scaling and Renormalization in two dimensional Quantum Gravity. Phys. Rev. D 2015, 92, 024026. [CrossRef]

58. Reuter, M.; Wetterich, C. Quantum Liouville field theory as solution of a flow equation. Nucl. Phys. B 1997, 506, 483-520. [CrossRef]

59. Reuter, M.; Saueressig, F. Fractal space-times under the microscope: A Renormalization Group view on Monte Carlo data. J. High Energy Phys. 2011, 2011, 012. [CrossRef]

60. Pawlowski, J.M. Aspects of the functional renormalisation group. Ann. Phys. 2007, 322, 2831-2915. [CrossRef]

61. Igarashi, Y.; Itoh, K.; Sonoda, H. Realization of Symmetry in the ERG Approach to Quantum Field Theory. Prog. Theor. Phys. Suppl. 2010, 181, 1-166. [CrossRef]

62. Pagani, C. Note on scaling arguments in the effective average action formalism. Phys. Rev. D 2016, 94, 045001. [CrossRef]

63. Pagani, C.; Sonoda, H. Products of composite operators in the exact renormalization group formalism. Prog. Theor. Exp. Phys. 2018, 2018, 023B02. [CrossRef]

64. Becker, M.; Pagani, C. Geometric Operators in Asymptotic Safety. Phys. Rev. D 2019, 99, 066002. [CrossRef]

65. Pagani, C. Functional Renormalization Group approach to the Kraichnan model. Phys. Rev. E 2015, 92, 033016. [CrossRef] [PubMed]

66. Reuter, M.; Saueressig, F. Quantum Gravity and the Functional Renormalization Group-The Road towards Asymptotic Safety; Cambridge University Press: Cambridge, UK, 2018.

67. Percacci, R. An Introduction to Covariant Quantum Gravity and Asymptotic Safety; World Scientific: Singapore, 2017.

68. Litim, D.F. Optimized renormalization group flows. Phys. Rev. D 2001, 64, 105007. [CrossRef]

69. Daum, J.E.; Reuter, M. Renormalization Group Flow of the Holst Action. Phys. Lett. B 2012, 710, $215-218$. [CrossRef]

70. Daum, J.E.; Reuter, M. Einstein-Cartan gravity, Asymptotic Safety, and the running Immirzi parameter. Ann. Phys. 2013, 334, 351-419. [CrossRef]

71. Pagani, C.; Percacci, R. Quantization and fixed points of non-integrable Weyl theory. Class. Quant. Grav. 2014, 31, 115005. [CrossRef]

72. Pagani, C.; Percacci, R. Quantum gravity with torsion and non-metricity. Class. Quant. Grav. 2015, 32, 195019. [CrossRef]

73. Reuter, M.; Schollmeyer, G.M. The metric on field space, functional renormalization, and metric-torsion quantum gravity. Ann. Phys. 2016, 367, 125-191. [CrossRef]

74. Manrique, E.; Rechenberger, S.; Saueressig, F. Asymptotically Safe Lorentzian Gravity. Phys. Rev. Lett. 2011, 106, 251302. [CrossRef] [PubMed]

75. Rechenberger, S.; Saueressig, F. A functional renormalization group equation for foliated spacetimes. J. High Energy Phys. 2013, 2013, 010. [CrossRef]

76. Biemans, J.; Platania, A.; Saueressig, F. Quantum gravity on foliated spacetimes: Asymptotically safe and sound. Phys. Rev. D 2017, 95, 086013. [CrossRef]

77. Biemans, J.; Platania, A.; Saueressig, F. Renormalization group fixed points of foliated gravity-matter systems. J. High Energy Phys. 2017, 2017, 093. [CrossRef]

78. Houthoff, W.B.; Kurov, A.; Saueressig, F. Impact of topology in foliated Quantum Einstein Gravity. Eur. Phys. J. C 2017, 77, 491. [CrossRef] [PubMed]

79. Tsamis, N.C.; Woodard, R.P. Physical Green's Functions in Quantum Gravity. Ann. Phys. 1992, 215, 96-155. [CrossRef]

80. Fröb, M.B. One-loop quantum gravitational corrections to the scalar two-point function at fixed geodesic distance. Class. Quant. Grav. 2018, 35, 035005. [CrossRef] 
81. Gray, A. The volume of a small geodesic ball of a Riemannian manifold. Mich. Math. J. 1974, 20, 329-344. [CrossRef]

82. Becker, D.; Reuter, M. Towards a C-function in 4D quantum gravity. J. High Energy Phys. 2015, $2015,065$. [CrossRef]

83. Ambjorn, J.; Goerlich, A.; Jurkiewicz, J.; Loll, R. Nonperturbative Quantum Gravity. Phys. Rept. 2012, 519, 127-210. [CrossRef] 